\title{
Acquired right-to-left shunt through an atrial septal perforation with cyanosis after percutaneous transvenous mitral commissurotomy
}

\author{
Masakazu Nakao, MD, ${ }^{a}$ Jack Kian Ch'ng, MD, ${ }^{\text {a }}$ Yoong Kong Kenny Sin, MBBS, FRCS, FAMS, ${ }^{a}$ Yeow Leng Chua, MBBS, \\ FRCS, FAMS, ${ }^{a}$ and Chung Yin Lee, MBBS, FAMS, ${ }^{\text {b }}$ Singapore
}

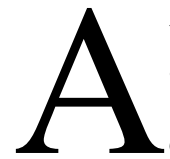

trial septal perforation (ASP) is a known complication after percutaneous transvenous mitral commissurotomy (PTMC), although it usually spontaneously heals. In cases in which ASP persists, the shunting is predominantly left to right, and rarely does the shunting reverse to cause clinical cyanosis.

\section{Clinical Summary}

A 49-year-old woman with a history of longstanding rheumatic heart disease presented with breathlessness and cyanosis, as well as bilateral lower limb swelling, for 2 weeks. She had undergone closed mitral valvotomy 26 years ago and subsequent PTMC twice 14 and 4 years ago, respectively. Other significant medical histories are atrial fibrillation and diabetes mellitus. Recent transthoracic echocardiography (TTE) 1 year ago showed moderate mitral stenosis (MS) with a mitral valve area of $1.3 \mathrm{~cm}^{2}$, mild mitral regurgitation (MR), severe tricuspid regurgitation (TR) with a pulmonary artery systolic pressure of $38 \mathrm{~mm} \mathrm{Hg}$, and a small atrial septal defect with left-to-right shunt, which was already shown on TTE 4 years ago after the second PTMC. Ejection fraction was 55\%. She had mild congestive cardiac failure (CCF) with multiple hospital admissions within the last few months.

She presented this admission with exacerbation of breathlessness and was found to be in CCF. She was centrally cyanosed, and oxygen saturation on room air was $80 \%$ to $86 \%$. TTE revealed right-toleft shunt through an ASP of $0.7 \mathrm{~cm}$ in diameter, severe MS, trivial MR, and severe TR with a dilated annulus (Figure 1).

Right heart study showed a mean right atrial pressure of $27 \mathrm{~mm}$ $\mathrm{Hg}$ and a pulmonary capillary wedge pressure of $22 \mathrm{~mm} \mathrm{Hg}$. Pulmonary artery systolic pressure was $51 \mathrm{~mm} \mathrm{Hg}$. The oximetric sampling showed $72 \%$ oxygen saturation in the left ventricle.

The patient improved with diuresis and subsequently agreed to surgical intervention.

Atrial septal repair with mitral valve replacement and tricuspid valve repair was performed with median sternotomy. On sternotomy, the right atrium was found to be enlarged and tense. Central

\footnotetext{
From the Departments of Cardiothoracic Surgery ${ }^{\mathrm{a}}$ and Cardiology, National Heart Centre, Singapore.

Received for publication Aug 30, 2007; accepted for publication Oct 19 , 2007.

Address for reprints: Masakazu Nakao, National Heart Center, Department of Cardiothoracic Surgery, Mistri Wing, 17 Third Hospital Ave, Singapore 168752 (E-mail: masakazu.nakao@nhc.com.sg).

J Thorac Cardiovasc Surg 2008;135:690-1

$0022-5223 / \$ 34.00$

Copyright (C) 2008 by The American Association of Thoracic Surgery doi:10.1016/j.jtcvs.2007.10.038
}

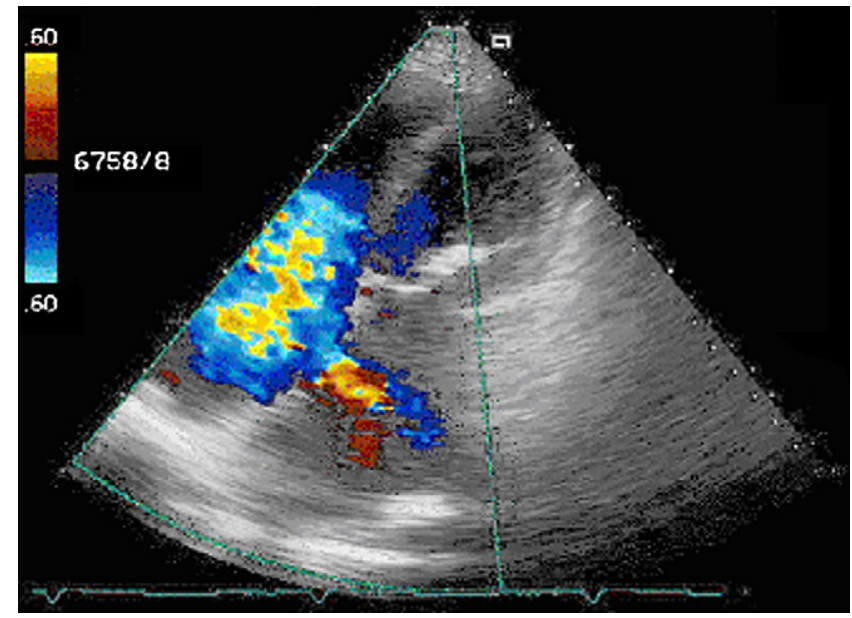

Figure 1. Preoperative transthoracic echocardiogram showing severe tricuspid regurgitation and right-to-left shunt.

venous pressure was $33 \mathrm{~mm} \mathrm{Hg}$. Cardiopulmonary bypass was instituted routinely, with aortic and bicaval cannulation. An ASP was confirmed in the central part of the interatrial septum. The mitral valve was exposed through a transseptal approach by enlarging the ASP; the valve and annulus were noted to be heavily calcified, with severe subvalvar involvement. The mitral valve was then replaced with a 29-mm Mosaic porcine bioprosthetic valve (Medtronic, Inc, Minneapolis, Minn). The interatrial septum was repaired. The tricuspid valve was then repaired with a 26-mm Edwards MC3 annuloplasty ring (Edwards LifeSciences, Irvine, Calif).

Postoperative TTE showed no residual shunt between the right and left atria. There was satisfactory bioprosthetic valve function with no MR, a pressure gradient of $4 \mathrm{~mm} \mathrm{Hg}$, and mild-to-moderate TR. Postoperative recovery was uncomplicated, and the patient was discharged on the 11th postoperative day.

\section{Discussion}

Inoue and colleagues ${ }^{1}$ reported PTMC in 1984 using an interatrial septal puncture. ASP is a well-known but rare complication of PTMC. Ishikura and associates ${ }^{2}$ reported that $15.2 \%$ of patients after PTMC were found to have an ASP 1 day after the procedure on TTE, and $4.3 \%$ of these persisted more than 1 year. However, none of these shunts were clinically significant. They were also normally left-to-right shunts and did not cause cyanosis. On rare occasions, however, the combination of MS, TR, and PHT can result in an right-to-left shunt, leading to cyanosis. ${ }^{3}$

Prior admissions for CCF showed a constant left-to-right shunt, which was noted on serial echocardiographic analysis for the past 4 
years. The patient had cyanosis on this admission in conjunction with recurrent CCF. TTE then revealed shunt reversal, explaining the onset of cyanosis.

One possible cause of shunt reversal was that the severe TR was directing the flow into the ASP, causing shunting to the left atrium as the severity increased. Another possibility was the change in the pressure gradient between the left and right atria: the pressure in the right atrium was $5 \mathrm{~mm} \mathrm{Hg}$ higher than the pulmonary capillary wedge pressure at the time of the right heart study. This might be due to the combination of worsening pulmonary hypertension and TR.

A reversal of shunt needs to be considered when there is a sudden onset of cyanosis during the follow-up after PTMC for MS. In such cases surgical correction should be considered in the treatment options, especially in the setting of significant valve disease.

\section{References}

1. Inoue K, Owaki T, Nakamura T, Kitamura F, Miyamoto N. Clinical application of transvenous mitral commissurotomy by a new balloon catheter. J Thorac Cardiovasc Surg. 1984;87:394-402.

2. Ishikura F, Nagata S, Yasuda S, Yamashita N, Miyatake K. Residual atrial septal perforation after percutaneous transvenous mitral commissurotomy with Inoue balloon catheter. Am Heart J. 1990;120:873-8.

3. Lai LP, Shyu KG, Hsu KL, Chiang FT, Tseng CD, Tseng YZ. Bidirectional shunt through a residual atrial septal defect after percutaneous transvenous mitral commissurotomy. Cardiology. 1993;83:205-7.

\title{
Successful lysis of an aortic prosthetic valve thrombosis with a dosing regimen for peripheral artery and bypass graft occlusions
}

\author{
Patricia K. Nguyen, MD, ${ }^{a, c}$ Scott M. Wasserman, MD, ${ }^{a, c}$ James I. Fann, MD, ${ }^{\text {b,d }}$ and John Giacomini, MD, \\ Stanford and Palo Alto, Calif
}

Video clips are available online.

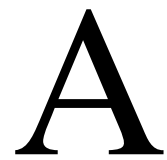

lthough valve thrombosis is infrequent, patients with prosthetic valves have a lifelong risk of this complication. Because operative mortality can be significant, thrombolysis has emerged as an alternative therapy for high-risk surgical patients. ${ }^{1-3}$ Recent reports have included the use of tissue-type plasminogen activator because of the unavailability of other agents ${ }^{4,5}$; however, the application of thrombolysis may be limited by increased risks of thrombus dislodgment, distal embolization, ${ }^{1}$ and hemorrhagic complications. ${ }^{3}$

Rapid infusion of thrombolytic agents has also been associated with higher complication rates. ${ }^{4,5}$ By determining the lowest effective infusion dosage, the infusion rate and duration can be set to minimize risks of thromboembolism and hemorrhage. To our knowledge, there are no data on the lowest effective dosing regimen

From the Department of Medicine, Division of Cardiology, ${ }^{\mathrm{a}}$ and the Department of Cardiothoracic Surgery, ${ }^{\text {, }}$ Stanford University, Stanford, Calif, and the Sections of Cardiology ${ }^{\mathrm{c}}$ and Cardiothoracic Surgery, ${ }^{\mathrm{d}}$ VA Palo Alto HCS, Palo Alto, Calif.

Received for publication Sept 13, 2007; accepted for publication Nov 15, 2007.

Address for reprints: John Giacomini, MD, Cardiovascular Medicine, Stanford University School of Medicine, 300 Pasteur Dr, Falk CVRB, Stanford, CA 94305 (E-mail: giacominijohn@yahoo.com).

J Thorac Cardiovasc Surg 2008;135:691-3

$0022-5223 / \$ 34.00$

Copyright (C) 2008 by The American Association for Thoracic Surgery doi:10.1016/j.jtcvs.2007.11.012 for specific patient subsets. It is likely that patients who are hemodynamically unstable require rapid lysis, and current recommended protocols $^{3}$ are indicated. For patients who are hemodynamically stable, however, a slower rate of infusion may be warranted. We present a case of prosthetic valve thrombosis successfully treated with a low-dose regimen of recombinant tissue-type plasminogen activator (rtPA, $1 \mathrm{mg} / \mathrm{h}$ without bolus), such as is typically used for peripheral artery bypass graft occlusions. Such an approach for treatment of prosthetic valve thrombosis has not been reported previously.

\section{Clinical Summary}

A 55-year-old woman with a mechanical aortic valve prosthesis (St Jude 19 mm; St Jude Medical Inc, Minneapolis, Minn) was seen for two days of exertional chest pain associated with nausea and diaphoresis. Two weeks before her admission, without seeking medical advice, the patient discontinued her warfarin sodium therapy. Physical examination revealed normal vital signs. Results of cardiopulmonary examination were unremarkable except for muffled aortic prosthetic valve sounds. Electrocardiography revealed normal sinus rhythm and left ventricular hypertrophy with strain.

Transthoracic echocardiography suggested restricted motion of aortic prosthetic leaflets, with turbulent flow on color flow Doppler scan. Continuous-wave Doppler echocardiography confirmed severe obstruction, with peak and mean gradients of $158 \mathrm{~mm} \mathrm{Hg}$ and $86 \mathrm{~mm}$ $\mathrm{Hg}$, respectively (Figure 1, A). There was mild aortic insufficiency. No obvious thrombus was seen. The left ventricle was normal in size, with normal systolic function and normal wall thickness. Fluoroscopy revealed decreased excursion of both aortic prosthetic leaflets (Figure 2, A; Video 1). The patient was admitted to the coronary care unit. Because of the patient's relative hemodynamic stability and the risk of reoperation, a trial of thrombolysis was elected. Both streptokinase and urokinase were unavailable at our institution and other nearby hospitals. We therefore chose a regimen of rtPA to achieve gradual lysis at the lowest effective dose and thus minimize 